\title{
Forcing tightness in products of fans
}

\author{
by
}

Jörg B r e n d l e (Tübingen and Hanover, N.H.) and Tim L a B er ge (Schenectady, N.Y., and DeKalb, Ill.)

\begin{abstract}
We prove two theorems that characterize tightness in certain products of fans in terms of families of integer-valued functions. We also define several notions of forcing that allow us to manipulate the structure of the set of functions from some cardinal $\theta$ to $\omega$, and hence, the tightness of these products. These results give new constructions of first countable $<\theta$-cwH spaces that are not $\leq \theta$-cwH.
\end{abstract}

1. Introduction. The $\theta-f a n F_{\theta}$ is the quotient space obtained by identifying the non-isolated points of the product $\theta \times(\omega+1)$ to a single point $\infty$. (Here $\theta$ has the discrete topology and $\omega+1$ has the order topology.) Thus, a neighborhood of $\infty$ is a set of the form

$$
V_{g}=\{\infty\} \cup\{\langle\alpha, m\rangle: m>g(\alpha)\}, \quad g \in \omega^{\theta} .
$$

When $\lambda \leq \theta$, we use sets

$$
\begin{aligned}
V_{g} \times U_{f}=\{(\infty, \infty)\} \cup\{(\langle\beta, m\rangle,\langle\alpha, n\rangle): m>g(\beta) \wedge n>f(\alpha)\}, \\
g \in \omega^{\theta}, f \in \omega^{\lambda},
\end{aligned}
$$

as a base at $(\infty, \infty)$ in the product $F_{\theta} \times F_{\lambda}$. (Technically, we should also include points with $\infty$ in one coordinate, but a result from [LL] says that it suffices to consider the subspace $\{(\infty, \infty)\} \cup(\theta \times \omega) \times(\lambda \times \omega)$.)

The tightness $t(p, X)$ of a point $p$ in a topological space $X$ is the supremum of the cardinalities of all $A \subseteq X$ such that $p \in \bar{A}$, but whenever $B \subseteq A$ and $|B|<|A|$, then $p \notin \bar{B}$. The tightness of $X$ is then $t(X)=\sup \{t(p, X)$ : $p \in X\}$. The tightness of $X$ is the least upper bound of the cardinalities of the subsets of $X$ needed to define the closure operator.

1991 Mathematics Subject Classification: Primary 04A20; Secondary 03E35, 54D15.

Research of the first author supported by DFG-grant Nr. Br 1420/1-1; the Emmy Noether Institute in Mathematics of Bar Ilan University, Israel; and the Minerva Foundation of Germany. Research of the second author partially supported by the Emmy Noether Institute in Mathematics of Bar Ilan University, Israel; the Minerva Foundation of Germany; and Union College. 
Clearly, the tightness of $F_{\theta}$ is $\omega$, and it is not hard to see that $t\left(F_{\theta} \times\right.$ $\left.F_{\lambda}\right)=t\left((\infty, \infty), F_{\theta} \times F_{\lambda}\right)$ (details are in [LL]). This motivates the following definition.

Definition 1.1. Let $\lambda \leq \theta$ be infinite cardinals and suppose that $A \subseteq$ $(\theta \times \omega) \times(\lambda \times \omega)$.

(1) If $\lambda<\theta$, we say that $A$ is $(\theta, \lambda)$-good if

(a) $(\infty, \infty) \in \bar{A}$;

(b) $\forall B \in A^{<\theta}((\infty, \infty) \notin \bar{B})$; and

(c) $\forall E \in[\lambda]^{<\lambda}((\infty, \infty) \notin \overline{A \cap((\theta \times \omega) \times(E \times \omega))})$.

(2) If $\lambda=\theta$, we say that $A$ is $(\theta, \theta)$-good if (a), (b), and the following are true:

(c) $\forall E \in[\theta]^{<\theta} \quad((\infty, \infty) \notin \overline{A \cap((\theta \times \omega) \times(E \times \omega))})$ and

(d) $\forall F \in[\theta]<\theta \quad((\infty, \infty) \notin \overline{A \cap((F \times \omega) \times(\theta \times \omega))})$.

The existence of a $(\theta, \lambda)$-good set $A$ implies that $t\left(F_{\theta} \times F_{\lambda}\right)=\theta$; moreover, if either $\theta^{\prime}<\theta$ and $\lambda^{\prime} \leq \lambda$ or $\theta^{\prime} \leq \theta$ and $\lambda^{\prime}<\lambda$, then $A$ cannot be construed as a subset of $F_{\theta^{\prime}} \times F_{\lambda^{\prime}}$. In [LL], it is shown that $\lambda^{\omega}<\theta$ implies that there are no $(\theta, \lambda)$-good sets; in particular, GCH implies that there are no $(\theta, \lambda)$-good sets whenever $\theta>\lambda \geq \operatorname{cf}(\lambda)>\omega$.

In this paper, we first prove two theorems that characterize the existence of certain good sets in terms of integer-valued functions (Section 2). We then give several forcing constructions of $(\theta, \lambda)$-good sets (Section 3). Starting with a regular cardinal $\theta$, the first construction gives a model with a $(\theta, \omega)$ good set but no $(\theta, \omega)$-gaps (this model has been obtained independently by Haim Judah $[\mathrm{J}])$. We then modify this construction to give a $(\theta, \omega)$-good set when $\theta$ is a singular cardinal of uncountable cofinality. The final construction gives models with $(\theta, \lambda)$-good sets when $\operatorname{cf}(\theta) \geq \omega_{1}$ and either $\lambda$ is regular and $\lambda \leq \theta$ or $\lambda^{\omega} \leq \theta$.

For the reader's convenience, we have collected the topological consequences of these results in Section 4 and provided a list of interesting open questions in Section 5.

2. Characterizing good sets. In this section, we prove two theorems that characterize the existence of certain good sets in terms of integer-valued functions. These theorems will use the following relations $\leq^{+}$and $\leq^{*}$ on $\omega^{\lambda}$ that generalize the usual notion of $\leq^{*}$ on $\omega^{\omega}$.

Definition 2.1. Let $\lambda$ be an infinite cardinal, and let $f, g \in \omega^{\lambda}$.

(a) We say $f \leq^{*} g$ if for all but finitely many $\alpha<\lambda, f(\alpha) \leq g(\alpha)$.

(b) We say $f \leq^{+} g$ if there is a $k \in \omega$ such that for all $\alpha<\lambda$, either $f(\alpha) \leq g(\alpha)$ or $f(\alpha) \leq k$. 
Notice that $\leq^{+}$is a reflexive, transitive relation on $\omega^{\lambda}$. We put $f={ }^{+} g$ if there is a $k \in \omega$ such that for all $\alpha \in \lambda$, either $f(\alpha)=g(\alpha)$ or both $g(\alpha), f(\alpha) \leq k$. This determines an equivalence relation on $\omega^{\lambda}$, and the order on these equivalence classes induced by $\leq^{+}$is a partial order. If $\lambda=\omega$ and we restrict ourselves to strictly increasing functions, then the two notions $\leq^{*}$ and $\leq^{+}$coincide. Also note that $f \leq^{*} g$ always implies $f \leq^{+} g$.

The following lemma gives a canonical way to construct a $(\theta, \lambda)$-good set. Whenever we refer to a family of functions from some set $A$ into $\omega$ as being bounded or unbounded, we mean with respect to the obvious $\leq^{+}$order on $\omega^{A}$ (unless we state explicitly $\leq^{*}$ ). When $\lambda=\omega$, the two notions of being bounded or unbounded coincide.

Lemma 2.2. Let $\lambda \leq \theta$ be infinite cardinals. Assume there is $\mathcal{F}=\left\{f_{\beta}\right.$ : $\beta<\theta\} \subseteq \omega^{\lambda}$ so that:

(a) $\mathcal{F}$ is unbounded;

(b) each $\mathcal{G} \in[\mathcal{F}]^{<\theta}$ is bounded;

(c) for all $E \in[\lambda]^{<\lambda}, \mathcal{F}\left\lceil E=\left\{f_{\beta}\lceil E: \beta<\theta\}\right.\right.$ is bounded.

Then there is a $(\theta, \lambda)$-good set.

Proof. Put $A=\left\{(\langle\beta, m\rangle,\langle\alpha, n\rangle): m, n \leq f_{\beta}(\alpha)\right\}$. We show:

(I) $(\infty, \infty) \in \bar{A}$;

(II) $(\infty, \infty) \notin \bar{B}$ for all $B \in[A]^{<\theta}$;

(III) $(\infty, \infty) \notin \bar{C}$ whenever $C=A \cap((\theta \times \omega) \times(E \times \omega))$ and $E \in[\lambda]^{<\lambda}$.

(I) Choose $g \in \omega^{\theta}$ and $f \in \omega^{\lambda}$. We have to show that $A$ intersects $V_{g} \times U_{f}$. By (a), we can choose $\beta<\theta$ so that $f_{\beta} \mathbb{Z}^{+} f$. That is, for every $k \in \omega$, there is an $\alpha_{k}<\lambda$ such that $f_{\beta}\left(\alpha_{k}\right)>f\left(\alpha_{k}\right)$ and $f_{\beta}\left(\alpha_{k}\right)>k$. Choose $k$ so that $k \geq g(\beta)$. Then $\left(\left\langle\beta, f_{\beta}\left(\alpha_{k}\right)\right\rangle,\left\langle\alpha, f_{\beta}\left(\alpha_{k}\right)\right\rangle\right) \in A \cap\left(V_{g} \times U_{f}\right)$.

(II) Let $B \in[A]^{<\theta}$; let $G=\{\beta<\theta: \exists m, n<\omega \exists \alpha<\lambda((\langle\beta, m\rangle$, $\langle\alpha, n\rangle) \in B)\}$. Then $G \in[\theta]^{<\theta}$. By assumption (b), there is an $f \in \omega^{\lambda}$ that is a $\leq^{+}$-bound for $\mathcal{G}=\left\{f_{\beta}: \beta \in G\right\}$. Thus for all $\beta \in G$, there is a $k_{\beta} \in \omega$ such that for each $\alpha<\lambda$, either $f_{\beta}(\alpha) \leq k_{\beta}$ or $f_{\beta}(\alpha) \leq f(\alpha)$. Define $g: \theta \rightarrow \omega$ by $g(\beta)=k_{\beta}$ for $\beta \in G, g(\beta)=0$ otherwise. We have to show that $B \cap\left(V_{g} \times U_{f}\right)=\emptyset$. To see this take $(\langle\beta, m\rangle,\langle\alpha, n\rangle) \in B$. Then both $m$ and $n$ are less than or equal to $f_{\beta}(\alpha)$; thus either $n \leq f_{\beta}(\alpha) \leq f(\alpha)$ and $\langle\alpha, n\rangle \notin U_{f}$ or $m \leq f_{\beta}(\alpha) \leq k_{\beta}=g(\beta)$ and $\langle\beta, m\rangle \notin V_{g}$.

(III) Similar; using (c) in place of (b).

We also need to recall some facts about $(\theta, \lambda)$-good sets from [LL]. Given a set $A \subseteq(\theta \times \omega) \times(\lambda \times \omega)$, and ordinals $\beta<\theta$ and $\alpha<\lambda$, we define $H_{\beta \alpha}(A)=H_{\beta \alpha}=\{(m, n): m, n \in \omega$ and $(\langle\beta, m\rangle,\langle\alpha, n\rangle) \in A\}$. We say $H_{\beta \alpha}$ is closed downward (abbreviated $\mathrm{cdw}$ ) if whenever $(m, n) \in H_{\beta \alpha}, n^{\prime} \leq n$, 
and $m^{\prime} \leq m$, then $\left(m^{\prime}, n^{\prime}\right) \in H_{\beta \alpha}$. The proofs of the following lemmas can be found in [LL].

Lemma 2.3. Suppose there is a $(\theta, \lambda)$-good set. Then there is a $(\theta, \lambda)$-good set $A$ such that each $H_{\beta \alpha}$ is finite and $c d w$.

Lemma 2.4. Suppose $A \subseteq(\theta \times \omega) \times(\lambda \times \omega)$ and that each $H_{\beta \alpha}$ is finite and cdw. Then $A$ accumulates at $(\infty, \infty)$ in $F_{\theta} \times F_{\lambda}$ if and only if

$$
\forall f: \lambda \rightarrow \omega \exists \beta<\theta \forall m \in \omega \exists \alpha<\lambda((\langle\beta, m\rangle,\langle\alpha, f(\alpha)\rangle) \in A) .
$$

In [G], Gruenhage showed that if there is a $(\theta, \omega)$-good set, then $\theta \geq \mathfrak{b}$.

THEOREM 2.5. The following are equivalent:

(a) there is an $\mathcal{F} \subseteq \omega^{\omega}$ of cardinality $\theta$ such that $\mathcal{F}$ is $\leq^{*}$-unbounded, but every $\mathcal{G} \in[\mathcal{F}]^{<\theta}$ is $\leq^{*}$-bounded;

(b) there is a $(\theta, \omega)$-good set.

Proof. (a) $\Rightarrow$ (b). By Lemma 2.2 .

(b) $\Rightarrow(\mathrm{a})$. Suppose that $A$ is a $(\theta, \omega)$-good set. Without loss of generality, we can assume that each $H_{\beta k}=H_{\beta k}(A)$ is finite and cdw. By Gruenhage's result, $\mathfrak{b} \leq \theta$; so let $\left\{g_{\alpha}: \alpha<\mathfrak{b}\right\} \subseteq \omega^{\omega}$ be an unbounded family of strictly increasing functions. For $\beta<\theta$ and $\alpha<\mathfrak{b}$, define a function $f_{\beta, \alpha}: \omega \rightarrow \omega$ by

$$
f_{\beta, \alpha}(k)=\left\{\begin{array}{l}
\max \left\{n: \exists m\left(g_{\alpha}(m) \geq k \wedge(\langle\beta, m\rangle,\langle k, n\rangle) \in A\right)\right\}, \\
0 \quad \text { if the above set is empty. }
\end{array}\right.
$$

Set $\mathcal{F}=\left\{f_{\beta, \alpha}: \beta<\theta\right.$ and $\left.\alpha<\mathfrak{b}\right\}$; we check that $(\mathrm{I}) \mathcal{F}$ is $<^{*}$-unbounded and (II) every $\mathcal{G} \in[\mathcal{F}]^{<\theta}$ is $<^{*}$-bounded.

(I) Let $f \in \omega^{\omega}$. Using Lemma 2.4 and the fact that $A$ is $(\theta, \omega)$-good, there is a $\beta<\theta$ such that for each $m \in \omega$, there is a $k_{m} \in \omega$ such that $\left(\langle\beta, m\rangle,\left\langle k_{m}, f\left(k_{m}\right)\right\rangle\right) \in A$. Define $g \in \omega^{\omega}$ by $g(m)=k_{m}$; then $g$ is finite-toone. Because the $g_{\alpha}$ 's are $\leq^{*}$-unbounded, there is an $\alpha<\mathfrak{b}$ such that for infinitely many $m, g_{\alpha}(m) \geq g(m)$. Then for each such $m, f_{\beta, \alpha}\left(k_{m}\right) \geq f\left(k_{m}\right)$, so that $f$ is not a $\leq^{*}$-bound for $\mathcal{F}$.

(II) Let $\mathcal{G} \in[\overline{\mathcal{F}}]^{<\theta}$. Set $G=\left\{\beta<\theta: \exists \alpha<\mathfrak{b}\left(f_{\beta, \alpha} \in \mathcal{G}\right)\right\}$. Set $B=$ $A \cap(G \times \omega) \times(\omega \times \omega)$; then $(\infty, \infty)$ is not in the closure of $B$. Choose $g \in \omega^{\theta}$ and $f \in \omega^{\omega}$ so that $B \cap\left(V_{g} \times U_{f}\right)=\emptyset$. We claim that $f$ is a $\leq^{*}$-bound for $\mathcal{G}$. Fix an $f_{\beta, \alpha} \in \mathcal{G}$. Now, $\beta \in G$, so $(\langle\beta, m\rangle,\langle k, n\rangle) \notin A$ whenever $m>g(\beta)$ and $n>f(k)$. Stated contrapositively, if $(\langle\beta, m\rangle,\langle k, n\rangle)$ is in $A$, then either $m \leq g(\beta)$ or $n \leq f(k)$. Take $k>g_{\alpha}(g(\beta))$ and any $m$ such that $g_{\alpha}(m) \geq k$; then $g_{\alpha}(m)>g_{\alpha}(g(\beta))$. Because $g_{\alpha}$ is strictly increasing, $m>g(\beta)$. Thus, if $n$ is such that $(\langle\beta, m\rangle,\langle k, n\rangle) \in A$, we must have $n \leq f(k)$. Taking the maximum over all such $n$ gives $f_{\beta, \alpha}(k) \leq f(k)$. Thus, whenever $k>g_{\alpha}(g(\beta))$, we have $f_{\beta, \alpha}(k) \leq f(k)$, whence $f_{\beta, \alpha} \leq^{*} f$. 
We now provide a consistent characterization of the existence of $(\theta, \theta)$ good sets in terms of families of integer-valued functions. The set-theoretic conditions we require in order to obtain this characterization are true in the models obtained by the Levy or Mitchell collapse of a large cardinal to $\omega_{2}$ and when $\mathrm{PFA}^{+}$holds, so this characterization may be useful in showing the consistency (relative to a large cardinal) of "there are no $\left(\omega_{2}, \omega_{2}\right)$-good sets".

Recall $\operatorname{cov}(\omega, \theta)=\min \left\{|\mathcal{C}|: \mathcal{C} \subseteq[\theta]^{\omega} \wedge \forall B \in[\theta]^{\omega} \exists C \in \mathcal{C}(B \subseteq C)\right\}$. It is well known that for all $n \in \omega, \operatorname{cov}\left(\omega, \omega_{n}\right)=\omega_{n}$ and that $\operatorname{cov}(\omega, \theta)>\theta$ for $\theta$ a cardinal of uncountable cofinality implies there is an inner model with large cardinals.

Theorem 2.6. Assume $\mathfrak{b} \leq \theta$ and $\operatorname{cov}(\omega, \theta) \leq \theta$. Then the following are equivalent:

(i) there is a $(\theta, \theta)$-good set;

(ii) there is an unbounded family $\mathcal{F}=\left\{f_{\beta}: \beta<\theta\right\} \subseteq \omega^{\theta}$ such that every $\mathcal{G} \in[\mathcal{F}]^{<\theta}$ is bounded and for every $B \in[\theta]^{<\theta}, \mathcal{F}\left\lceil B=\left\{f_{\beta}\lceil B: \beta<\theta\}\right.\right.$ is bounded.

Proof. (ii) $\Rightarrow$ (i). This follows from Lemma 2.2 .

(i) $\Rightarrow\left(\right.$ ii). Let $A$ be a $(\theta, \theta)$-good set. We can assume that each $H_{\beta \alpha}$ (defined just before Lemma 2.3) is symmetric, i.e., $H_{\beta \alpha}=\{(n, m):(m, n) \in$ $\left.H_{\alpha \beta}\right\}$. Let $\left\{B_{\delta}: \delta<\operatorname{cov}(\omega, \theta)\right\}$ enumerate a covering family; for $\delta<$ $\operatorname{cov}(\omega, \theta)$, let $r_{\delta}: \omega \rightarrow B_{\delta}$ be a bijection. Let $\left\{g_{\gamma}: \gamma<\mathfrak{b}\right\} \subseteq \omega^{\omega}$ be an unbounded family of strictly increasing functions.

For $\beta<\theta, \delta<\operatorname{cov}(\omega, \theta)$, and $\gamma<\mathfrak{b}$, define a function $f_{\beta \delta \gamma}: \theta \rightarrow \omega$ by

$$
f_{\beta \delta \gamma}(\alpha)=\left\{\begin{array}{l}
\max \left\{n: \exists m\left(g_{\gamma}(m) \geq r_{\delta}^{-1}(\alpha) \wedge(\langle\beta, m\rangle,\langle\alpha, n\rangle) \in A\right)\right\}, \\
0 \quad \text { if the above set is empty or } \alpha \notin B_{\delta} .
\end{array}\right.
$$

Set $\mathcal{F}=\left\{f_{\beta \delta \gamma}: \beta<\theta, \delta<\operatorname{cov}(\omega, \theta)\right.$, and $\left.\gamma<\mathfrak{b}\right\}$. We claim

(I) $\mathcal{F}$ is unbounded;

(II) whenever $B \in[\theta]<\theta$, then $\mathcal{F}\lceil B$ is bounded; and

(III) whenever $\mathcal{G} \in[\mathcal{F}]^{<\theta}$, then $\mathcal{G}$ is bounded.

The proofs of (II) and (III) are similar to those for Theorem 2.5. For example, to prove (II), fix a $B \in[\theta]^{<\theta}$, and set $A^{\prime}=A \cap((\theta \times \omega) \times(B \times \omega))$. Then $(\infty, \infty) \notin \overline{A^{\prime}}$, so there is a $g \in \omega^{\theta}$ so that $A^{\prime} \cap\left(V_{g} \times U_{g}\right)=\emptyset$. We claim that $g$ is a bound for $\mathcal{F}\lceil B$.

To see this, fix $\beta<\theta, \delta<\operatorname{cov}(\omega, \theta)$, and $\gamma<\mathfrak{b}$. Take any $\alpha \in B_{\delta} \backslash$ $r_{\delta}\left(\left\{0,1, \ldots, g_{\gamma}(g(\beta))\right\}\right)$ and any $m$ with $g_{\gamma}(m) \geq r_{\delta}^{-1}(\alpha)$; then $g_{\gamma}(m)>$ $g_{\gamma}(g(\beta))$. Because $g_{\gamma}$ is increasing, $m>g(\beta)$. Thus, if $(\langle\beta, m\rangle,\langle\alpha, n\rangle) \in$ $A^{\prime}$, we must have $n \leq g(\alpha)$. Taking the maximum over all such $n$ yields $f_{\beta \delta \gamma}(\alpha) \leq g(\alpha)$, so clearly $f_{\beta \delta \gamma} \leq^{*} g$. The proof of (III) is similar. 
To show that (I) is true, we need the following:

Claim. For every $f \in \omega^{\theta}$, there is $a \beta<\theta$ and a sequence $\left\{\alpha_{m}: m \in \omega\right\}$ so that whenever $m \in \omega$, either $f\left(\alpha_{m}\right) \geq m$ and $\left(\langle\beta, m\rangle,\left\langle\alpha_{m}, f\left(\alpha_{m}\right)\right\rangle\right) \in A$, or $f\left(\alpha_{m}\right)<m$ and $\left(\langle\beta, m\rangle,\left\langle\alpha_{m}, m\right\rangle\right) \in A$.

Suppose otherwise. Then there is an $f \in \omega^{\theta}$ so that for all $\beta<\theta$, there is an $m_{\beta} \in \omega$ such that for all $\alpha<\theta$,

$(*)\left(\left\langle\beta, m_{\beta}\right\rangle,\left\langle\alpha, m_{\beta}\right\rangle\right) \in A \Rightarrow f(\alpha) \geq m_{\beta}$ and

$(* *)\left(\left\langle\beta, m_{\beta}\right\rangle,\langle\alpha, f(\alpha)\rangle\right) \in A \Rightarrow f(\alpha)<m_{\beta}$.

Define $g \in \omega^{\theta}$ by $g(\beta)=\max \left\{f(\beta), m_{\beta}\right\}+1$. Because $(\infty, \infty) \in \bar{A}$, there are $\alpha<\beta<\theta$ with $(\langle\beta, g(\beta)\rangle,\langle\alpha, g(\alpha)\rangle) \in A$. Now, $H_{\beta \alpha}$ is cdw, so $\left(\left\langle\beta, m_{\beta}\right\rangle,\langle\alpha, f(\alpha)\rangle\right) \in A$; by $(* *), f(\alpha)<m_{\beta}$. By symmetry, $f(\beta)<m_{\alpha}$. Suppose that $m_{\beta} \leq m_{\alpha}$ (the other case is dual). Then $\left(\left\langle\beta, m_{\beta}\right\rangle,\left\langle\alpha, m_{\beta}\right\rangle\right) \in$ $A$. By $(*), f(\alpha) \geq m_{\beta}$, a contradiction. This proves the claim.

To finish the proof of (I), fix $f \in \omega^{\theta}$, and take $\beta<\theta$ and $\left\{\alpha_{m}: m \in \omega\right\}$ as in the claim. Find a $\delta<\operatorname{cov}(\omega, \theta)$ so that $\left\{\alpha_{m}: m \in \omega\right\} \subseteq B_{\delta}$. Define $h \in \omega^{\omega}$ by $h(m)=r_{\delta}^{-1}\left(\alpha_{m}\right)$; then $h$ is finite-to-one. Find a $\gamma<\mathfrak{b}$ so that for infinitely many $m, h(m) \leq g_{\gamma}(m)$. Then for such an $m$, either $f\left(\alpha_{m}\right) \geq m$ and $f_{\beta \delta \gamma}\left(\alpha_{m}\right) \geq f\left(\alpha_{m}\right)$, or $f\left(\alpha_{m}\right)<m$ and $f_{\beta \delta \gamma}\left(\alpha_{m}\right) \geq m$, so that $f_{\beta \delta \gamma} \mathbb{E}^{+} f$.

3. Forcing good sets. It is a well known fact, due independently to Hausdorff $[\mathrm{H}]$ and Rothberger $[\mathrm{R}]$, that the existence of a $(\theta, \omega)$-gap in $\left(\omega^{\omega}, \leq^{*}\right)$ is equivalent to the existence of a well-ordered unbounded sequence of order type $\theta$ in $\left(\omega^{\omega}, \leq^{*}\right)$. This means that the existence of a $(\theta, \omega)$-gap implies the existence of a $(\theta, \omega)$-good set. It is certainly consistent that the converse is true (consider, e.g., a model of $\mathrm{CH}$ ), so it is natural to ask if the converse is true in ZFC. In fact, when $\theta \geq \omega_{2}$ and $\operatorname{cf}(\theta) \geq \omega_{1}$, there is a model in which there is a $(\theta, \omega)$-good set, but only $\left(\omega_{1}, \omega_{1}\right)$ - and $\left(\omega_{1}, \omega\right)$-gaps.

Before we define the partial orders that give these models, we state some useful lemmas about product forcing. If $\mathcal{F} \subseteq \omega^{\omega}$ and $g \in \omega^{\omega}$, we say that $g>^{*} \mathcal{F}$ if $g>^{*} f$ for all $f \in \mathcal{F}$. We say that a real $f$ in a universe larger than $V$ is unbounded over $V$ if $f \mathbb{Z}^{*} g$ for all $g \in \omega^{\omega} \cap V$.

Lemma 3.1. Let $\mathbb{P}$ and $\mathbb{Q}$ be partial orders. Suppose $\dot{f}$ is a $\mathbb{P}$-name for a real and $\dot{g}$ is a $\mathbb{Q}$-name for a real. If $\Vdash_{\mathbb{P}}$ " $\dot{f}$ is unbounded over $V$ ", then $\Vdash_{\mathbb{P} \times \mathbb{Q}}$ “ $\neg(\dot{f} \leq * \dot{g}) "$.

Proof. Fix $n \in \omega$ and $(p, q) \in \mathbb{P} \times \mathbb{Q}$. Because $\Vdash_{\mathbb{P}}$ " $\dot{f}$ is unbounded over $V "$, there is an $l \geq n$ such that $\forall m \in \omega\left(\neg\left(p \Vdash_{\mathbb{P}}\right.\right.$ " $\dot{f}(l) \leq m$ " $\left.)\right)$.

Choose a $q^{\prime} \leq q$ and an $m \in \omega$ so that $q^{\prime} \Vdash_{\mathbb{Q}} " \dot{g}(l)=m$ ". Then we can find a $p^{\prime} \leq p$ and a $k>m$ such that $p^{\prime} \Vdash_{\mathbb{P}} " \dot{f}(l)=k "$. Thus, $\left(p^{\prime}, q^{\prime}\right) \Vdash_{\mathbb{P} \times \mathbb{Q}}$ " $\dot{f}(l)>\dot{g}(l) "$. 
LEMMA 3.2. Suppose that $\dot{h}_{1}$ and $\dot{h}_{2}$ are $\mathbb{P}$-names for reals, $\dot{g}$ is a $\mathbb{Q}$-name for a real, and $(p, q) \Vdash_{\mathbb{P} \times \mathbb{Q}}$ " $\dot{h}_{1}<^{*} \dot{g}<^{*} \dot{h}_{2}$ ". Then $p \Vdash_{\mathbb{P}} " \exists j \in \omega^{\omega} \cap V\left(\dot{h}_{1}<^{*}\right.$ $\left.j<* \dot{h}_{2}\right) "$.

Proof. Without loss of generality, we assume that for some $k^{\prime} \in \omega$,

$$
(p, q) \Vdash_{\mathbb{P} \times \mathbb{Q}} \text { " } \forall n \geq k^{\prime}\left(\dot{h}_{1}(n)<^{*} \dot{g}(n)<{ }^{*} \dot{h}_{2}(n)\right) " .
$$

For each $n \in \omega$, define $j(n)$ by taking a $q^{\prime} \leq q$ and an $m \in \omega$ so that $q^{\prime} \Vdash_{\mathbb{Q}} " \dot{g}(n)=m "$, and setting $j(n)=m$.

We claim that $p \Vdash_{\mathbb{P}}$ " $\dot{h}_{1}<^{*} j<^{*} \dot{h}_{2}$ ". Otherwise, for each $k \in \omega$, there is an $n \geq k$ and a $p^{\prime} \leq p$ such that $p^{\prime} \Vdash_{\mathbb{P}} " \neg\left(\dot{h}_{1}(n)<j(n)<\dot{h}_{2}(n)\right)$ ". Fix such a $p^{\prime}$ and $n$ for the $k^{\prime}$ given above, and find a $q^{\prime} \leq q$ such that $q^{\prime} \Vdash_{\mathbb{Q}} " \dot{g}(n)=j(n) "$. Then

$$
\begin{aligned}
\left(p^{\prime}, q^{\prime}\right) \Vdash_{\mathbb{P} \times \mathbb{Q}} " & \left(\dot{h}_{1}(n)<\dot{g}(n)<\dot{h}_{2}(n)\right) \\
& \wedge \neg\left(\dot{h}_{1}(n)<j(n)<\dot{h}_{2}(n)\right) \wedge(\dot{g}(n)=j(n)),,
\end{aligned}
$$

a contradiction.

For a set $A, \mathbb{C}_{A}$ is the partial order $\operatorname{Fn}(A \times \omega, \omega)=\{p: p$ is a finite partial function from $A \times \omega$ into $\omega\} . V_{A}$ is the generic extension of $V$ by $\mathbb{C}_{A}$. We will need the fact, due to Kunen $[\mathrm{Ku}]$, that forcing with $\mathbb{C}_{A}$ over a model of $\mathrm{CH}$ does not add an $\omega_{2}$-sequence in $\left(\omega^{\omega}, \leq^{*}\right)$.

Lemma 3.3. Assume $C H$ and set $\theta=\omega_{2}$. In $V_{\theta}$, let $\mathbb{P}=\prod_{\alpha<\theta} \mathbb{P}_{\alpha}$ be a ccc finite support product of $\aleph_{1}$-sized partial orders such that $\prod_{\alpha<\beta} \mathbb{P}_{\alpha} \in V_{\beta}$. Set $\mathbb{Q}=\mathbb{C}_{\theta} \star \dot{\mathbb{P}}$, and let $H$ be $\mathbb{Q}$-generic over $V$. Then there are no well-ordered $\omega_{2}$-sequences in $V[H]$.

Proof. By way of contradiction, let $\left\{\dot{f}_{\alpha}: \alpha<\omega_{2}\right\}$ be a collection of $\mathbb{Q}$-names for a well-ordered $\omega_{2}$-sequence. Without loss of generality, each $\dot{f}_{\alpha}=\bigcup_{n, m \in \omega}\{(m, n)\} \times A_{m n}$, where $A_{m n}$ is a maximal countable antichain and each $q \in A_{m n}$ has the form $q=\left(c,\left\langle\dot{p}_{\alpha_{1}}, \ldots, \dot{p}_{\alpha_{k}}\right\rangle\right)$, where $c \in \mathbb{C}_{\theta}$ and $\dot{p}_{\alpha_{i}} \in \dot{\mathbb{P}}_{\alpha_{i}}$. That is, because the supports of conditions in $\dot{\mathbb{P}}$ are finite, we can assume that the $\mathbb{C}_{\theta}$ part of a condition is strong enough to decide the support of the $\dot{\mathbb{P}}$ part.

Define $\operatorname{suppt}(q)=\left\{\alpha_{1}, \ldots, \alpha_{k}\right\}$ and for each $\alpha \in \omega_{2}$,

$$
A_{\alpha}=\operatorname{suppt}\left(\dot{f}_{\alpha}\right)=\bigcup_{\substack{m, n \in \omega \\ q \in A_{m n}}} \operatorname{suppt}(q) .
$$

By thinning and re-indexing, we can assume that the $A_{\alpha}$ 's are a delta system with root $\Delta$ and that $\alpha<\alpha^{\prime}<\omega_{2}$ implies

$$
\max \Delta<\min \left(A_{\alpha} \backslash \Delta\right) \leq \max \left(A_{\alpha} \backslash \Delta\right)<\min \left(A_{\alpha}^{\prime} \backslash \Delta\right) .
$$


Set $\beta=\max (\Delta)+1$, and force with $\mathbb{C}_{\beta} \star \prod_{\alpha<\beta} \dot{\mathbb{P}}_{\alpha}$. Notice that $\mathrm{CH}$ is still true, so if we force with $\mathbb{C}_{[\beta, \theta)}$, we obtain a model $V^{\prime}$ with no $\omega_{2}$-chains. We can also assume that each $\dot{f}_{\alpha}$ is a $\prod_{\xi \in A_{\alpha} \backslash \Delta} \mathbb{P}_{\xi}$-name.

Set $E=\left\{\xi \in \omega_{2} \backslash \beta\right.$ : for some even $\left.\alpha, \xi \in A_{\alpha} \backslash \Delta\right\}$, and set $O=$ $\omega_{2} \backslash(E \cup \beta)$. Finally, let $\mathbb{P}_{E}=\prod_{\xi \in E} \mathbb{P}_{\xi}$ and $\mathbb{P}_{O}=\prod_{\xi \in O} \mathbb{P}_{\xi}$.

Working in $V^{\prime}$, fix a condition $(p, q) \in \mathbb{P}_{E} \times \mathbb{P}_{O}$ such that

$$
(p, q) \Vdash_{\mathbb{P}_{E} \times \mathbb{P}_{O}} \text { " } \forall \alpha<\omega_{2}\left(\alpha \text { even implies } \dot{f}_{\alpha}<^{*} \dot{f}_{\alpha+1}<^{*} \dot{f}_{\alpha+2}\right) \text { ". }
$$

By Lemma 3.2, for each even $\alpha<\omega_{2}$ we can find a $p_{\alpha} \leq p$ and a $j_{\alpha} \in \omega^{\omega} \cap V^{\prime}$ such that

$$
p_{\alpha} \Vdash_{\mathbb{P}} " \dot{f}_{\alpha}<{ }^{*} j_{\alpha}<\dot{f}_{\alpha+2} " .
$$

Now, because supports are finite and each $\left|\mathbb{P}_{\alpha}\right| \leq \omega_{1}$, we can find an $A \in$ $\left[\omega_{2}\right]^{\omega_{2}}$ so that whenever $\alpha, \alpha^{\prime} \in A$, then $p_{\alpha}$ and $p_{\alpha^{\prime}}$ are compatible. But then $\alpha<\alpha^{\prime} \in A$ implies that $j_{\alpha}<^{*} j_{\alpha^{\prime}}$, contradicting the fact that there are no $\omega_{2}$-sequences in $V^{\prime}$.

Hechler forcing is the partial order $\mathbb{D}=\left\{(s, f): s \in \omega^{<\omega}, f \in \omega^{\omega}\right.$, and $s \subseteq f\}$, ordered so that $(s, f) \leq(t, g)$ if and only if $s \supseteq t$ and $\forall n \in \omega(f(n) \geq$ $g(n))$. Clearly, $\mathbb{D}$ is $\sigma$-centered and adds a real that eventually dominates all ground model reals. The following result has been obtained independently by Judah $[\mathrm{J}]$.

Theorem 3.4. Assume $V \vDash C H$ and set $\theta=\omega_{2}$. Then there is a partial order $\mathbb{Q}$ such that whenever $H$ is $\mathbb{Q}$-generic over $V$, then the following are true in $V[H]$ :

(1) There is a family $\mathcal{F}=\left\{f_{\alpha}: \alpha<\theta\right\} \subseteq \omega^{\omega}$ such that:

(a) for all $\beta<\theta,\left\{f_{\alpha}: \alpha<\beta\right\}$ is bounded;

(b) $\mathcal{F}$ is unbounded.

(2) There are no well-ordered sequences of length $\omega_{2}$ in $\left(\omega^{\omega}, \leq^{*}\right)$.

Pr o o f. We define $\mathbb{Q}$ as a two-step iteration. The first step of the iteration is simply $\mathbb{C}_{\theta}$. We define the second step by working in $V_{\theta}$. Let $\mathbb{P}_{\beta}$ be $\mathbb{D}^{V_{\beta}}$, i.e., Hechler forcing in the sense of the model obtained by adding the first $\beta$-many Cohen reals. In $V_{\theta}$, each $\mathbb{D}_{\beta}$ is $\sigma$-centered, so the finite support product $\mathbb{P}=\prod_{\beta<\theta} \mathbb{P}_{\beta}$ is ccc. Hence, $\mathbb{Q}=\mathbb{C}_{\theta} \star \mathbb{P}$ is ccc.

Let $H$ be $\mathbb{Q}$-generic; in $V[H]$, define $\mathcal{F}=\left\{f_{\beta}: \beta<\theta\right\}$, where $f_{\beta}$ is the $\beta$ th Cohen real. Let $g_{\beta}$ be the Hechler real added by $\mathbb{P}_{\beta}$ over $V_{\theta}$; because $\left\{f_{\alpha}: \alpha<\beta\right\} \subseteq V_{\beta}$, we have $f_{\alpha} \leq^{*} g_{\beta}$ for each $\alpha<\beta$. This establishes (a).

Let $\dot{g}$ be a $\mathbb{Q}$-name for a real. Note that for all $\beta<\theta$, we have

$$
\mathbb{Q} \cong\left[\left(\mathbb{C}_{\beta} \star \prod_{\alpha<\beta} \dot{\mathbb{P}}_{\alpha}\right) \times \mathbb{C}_{[\beta, \theta)}\right] \star \prod_{\alpha \geq \beta} \dot{\mathbb{P}}_{\alpha}
$$


By this observation and the fact that $\mathbb{Q}$ is ccc, there is a $\beta<\theta$ such that $\dot{g}$ is a $\mathbb{C}_{\beta} \star \prod_{\alpha<\beta} \mathbb{P}_{\alpha}$-name. Because $f_{\beta}$ is unbounded over $V$, Lemma 3.1 and the fact that $\leq^{*}$ is upwards absolute imply that $\Vdash_{\mathbb{Q}} " \dot{f}_{\beta} \mathbb{Z}^{*} \dot{g}$ ". Hence, no real in $V[H]$ bounds $\mathcal{F}$, so (b) is established.

Note that the partial order we have defined satisfies the hypotheses of Lemma 3.3, so (2) is also true.

We can also show, via a modification of the "isomorphism of names" argument originally due to Kunen $[\mathrm{Ku}]$, that the forcing construction given above yields a model with no $\omega_{2}$-sequences when $\theta$ is any cardinal (see $[\mathrm{Br}]$ for details). When $\theta$ is regular, this gives a model with a $(\theta, \omega)$-good set, but only $\left(\omega_{1}, \omega_{1}\right)$ - and $\left(\omega_{1}, \omega\right)$-gaps.

The above proof does not quite suffice to produce a $(\theta, \omega)$-good set when $\theta$ is a singular cardinal of uncountable cofinality, because we need to bound all small subfamilies of $\mathcal{F}$. Fortunately, we can use the ccc to accomplish this.

Theorem 3.5. Suppose $V \vDash G C H$ and that $\omega_{1} \leq \operatorname{cf}(\theta)<\theta$. Then there is a ccc partial order $\mathbb{Q}$ such that whenever $H$ is $\mathbb{Q}$-generic over $V$, there is a family $\mathcal{F}=\left\{f_{\alpha}: \alpha<\theta\right\} \subseteq \omega^{\omega}$ in $V[H]$ such that:

(1) $\mathcal{F}$ is unbounded;

(2) for all $\mathcal{G} \in[\mathcal{F}]^{<\theta}, \mathcal{G}$ is bounded.

Pro of. As before, $\mathbb{Q}$ will be a two-step iteration, with first step $\mathbb{C}_{\theta}$. Let $f_{\beta}$ be the $\beta$ th Cohen real, and set $\mathcal{F}=\left\{f_{\beta}: \beta<\theta\right\}$. The second step of the iteration is defined in $V_{\theta}$ as $\prod_{A \in[\theta]<\theta} \mathbb{P}_{A}$, where $\mathbb{P}_{A}=\mathbb{D}^{V_{A}}$, i.e., Hechler forcing in the sense of the model obtained by adding the Cohen reals with indices in $A$. We therefore have

$$
\mathbb{Q}=\mathbb{C}_{\theta} \star \prod_{A \in[\theta]<\theta} \dot{\mathbb{P}}_{A}
$$

Clearly, $\mathbb{Q}$ is ccc. As before, $\mathcal{F}$ remains unbounded in $V[H]$.

To see that every small family is bounded, take $B \in[\theta]^{<\theta} \cap V[H]$. Because $\mathbb{Q}$ is ccc, there is an $A \in[\theta]^{<\theta} \cap V$ such that $B \subseteq A$. Then the Hechler real added by $\mathbb{P}_{A}$ bounds $\left\{f_{\beta}: \beta \in B\right\}$.

Again it can be shown that the model does not contain well-ordered sequences of length $\omega_{2}$ in $\left(\omega^{\omega}, \leq^{*}\right)$.

We next describe notions of forcing for adding $(\theta, \lambda)$-good sets for some cardinals that satisfy $\omega_{1} \leq \lambda \leq \theta$. The method will be similar to that used above - but proving that our iteration is ccc will now be non-trivial.

TheOREM 3.6. Let $\lambda \leq \theta$ cardinals with $\operatorname{cf}(\theta) \geq \omega_{1}$. Assume either $\lambda$ is regular or $\lambda^{\omega} \leq \theta$. There is a ccc partial order $\mathbb{P}$ that adds a family $\mathcal{F}=\left\{f_{\xi}: \xi<\theta\right\} \subseteq \omega^{\lambda}$ satisfying: 
(a) $\mathcal{F}$ is $\leq^{+}$-unbounded;

(b) for all $B \subseteq \theta$ with $|B|<\theta,\left\{f_{\xi}: \xi \in B\right\}$ is $\leq^{*}$-bounded;

(c) for all $A \subseteq \lambda$ with $|A|<\lambda, \mathcal{F} \mid A=\left\{f_{\xi}\lceil A: \xi<\theta\}\right.$ is $\leq^{*}$-bounded.

P r o of. Let $V_{0}$ be the ground model. As before, $\mathbb{P} \in V_{0}$ will be a two-step iteration. To define the first step, we start with a function $H: \theta \rightarrow[\lambda]^{\omega}$ that satisfies either

(1) $\left.\forall A \in[\lambda]^{\omega}(\mid \xi<\theta: H(\xi)=A\} \mid=\theta\right)$

or (in case $\lambda$ is regular and $\lambda^{\omega} \leq \theta$ fails)

(2) $\forall \xi<\theta \forall \zeta<\lambda(\zeta \in H(\xi) \Rightarrow \zeta+1 \in H(\xi))$ and

(3) $\forall \zeta<\lambda(\operatorname{cf}(\zeta)=\omega \Rightarrow|\{\xi<\theta: \sup (H(\xi))=\zeta\}|=\theta)$.

We define $\mathbb{P}_{0}=\left\{c \in \mathbb{C}_{\theta \times \lambda}: \forall \xi<\theta \forall \zeta<\lambda((\xi, \zeta) \in \operatorname{dom}(c) \Rightarrow \zeta \in\right.$ $H(\xi))\}$, ordered by reverse containment (notice that our notation here is slightly different from that preceding Lemma 3.3: $\mathbb{C}_{A}$ denotes $\operatorname{Fn}(A, \omega)$ ). Obviously, $\mathbb{P}_{0}$ is forcing isomorphic to $\mathbb{C}_{\theta}$; we think of $h_{\xi}$ (the $\xi$ th Cohen real added by $\mathbb{P}_{0}$ ) as having domain $H(\xi)$. Extend $h_{\xi}$ to a function $f_{\xi} \in \omega^{\lambda}$ by setting $f_{\xi}(\zeta)=h_{\xi}(\zeta)$ for all $\zeta \in H(\xi)$ and $f_{\xi}(\zeta)=0$ for all $\zeta \notin H(\xi)$.

Let $V_{1}$ be the extension of $V_{0}$ by $\mathbb{P}_{0}$. In $V_{1}$, we define for each $A \subseteq \lambda$ with $|A|<\lambda$ and $A \in V_{0}$ and for each $B \subseteq \theta$ with $|B|<\theta$ and $B \in V_{0}$ partial orders $\mathbb{Q}_{A}$ and $\mathbb{R}_{B}$ as follows: $\mathbb{Q}_{A}=\left\{\langle s, F\rangle: s \in \mathbb{C}_{A} \wedge F \in[\theta]^{<\omega}\right\}$ ordered so that $\langle s, F\rangle \leq\left\langle s^{\prime}, F^{\prime}\right\rangle$ if and only if $s \supseteq s^{\prime}, F \supseteq F^{\prime}$ and

$$
\forall \xi \in F^{\prime} \forall \zeta \in \operatorname{dom}(s) \backslash \operatorname{dom}\left(s^{\prime}\right)\left(f_{\xi}(\zeta) \leq s(\zeta)\right) .
$$

Similarly, $\mathbb{R}_{B}=\left\{\langle t, G\rangle: t \in \mathbb{C}_{\lambda} \wedge G \in[B]^{<\omega}\right\}$, ordered in the same way as $\mathbb{Q}_{A}:\langle t, G\rangle \leq\left\langle t^{\prime}, G^{\prime}\right\rangle$ if and only if $t \supseteq t^{\prime}, G \supseteq G^{\prime}$ and

$$
\forall \xi \in G^{\prime} \forall \zeta \in \operatorname{dom}(t) \backslash \operatorname{dom}\left(t^{\prime}\right)\left(f_{\xi}(\zeta) \leq t(\zeta)\right) .
$$

In $V_{1}$, let $\mathbb{P}_{1}=\prod_{A} \mathbb{Q}_{A} \times \prod_{B} \mathbb{R}_{B}$ be the finite support product of the $\mathbb{Q}_{A}$ 's and $\mathbb{R}_{B}$ 's. In $V_{0}$, let $\mathbb{P}=\mathbb{P}_{0} \star \dot{\mathbb{P}}_{1}$. Also in $V_{0}$, let $\left\langle A_{\alpha}: \alpha<\lambda^{\prime}\right\rangle$ enumerate $[\lambda]^{<\lambda}$ and $\left\langle B_{\beta}: \beta<\theta^{\prime}\right\rangle$ enumerate $[\theta]^{<\theta}$.

Claim. $\mathbb{P}_{1}$ is ccc in $V_{1}$.

To prove the claim, it suffices to show that for every $A \in\left[\lambda^{\prime}\right]^{<\omega}$ and $B \in\left[\theta^{\prime}\right]^{<\omega}$, the partial order $\mathbb{P}_{0} \star\left(\prod_{\alpha \in A} \dot{\mathbb{Q}}_{A_{\alpha}} \times \prod_{\beta \in B} \dot{\mathbb{R}}_{B_{\beta}}\right)$ is ccc in $V_{0}$. For each $\gamma \in \omega_{1}$, fix a condition

$$
p^{\gamma}=\left\langle c^{\gamma},\left\langle\left\langle s_{\alpha}^{\gamma}, F_{\alpha}^{\gamma}\right\rangle: \alpha \in A\right\rangle,\left\langle\left\langle t_{\beta}^{\gamma}, G_{\beta}^{\gamma}\right\rangle: \beta \in B\right\rangle\right\rangle .
$$

We do not need to work with names because conditions are finite partial functions, so we can assume the $\mathbb{P}_{0}$ part of a condition is strong enough to decide the second part. For $c \in \mathbb{P}_{0}$, set $d(c)=\{\xi<\theta: \exists \zeta \in H(\xi)((\xi, \zeta) \in$ $\operatorname{dom}(c))\}$. 
By applying a delta-system argument, we can assume that there are $c$, $s_{\alpha}, F_{\alpha}, t_{\beta}$, and $G_{\beta}$ such that for all $\gamma<\omega_{1}$, all $\alpha \in A$, and all $\beta \in B$,

$$
p^{\gamma}=\left\langle c \cup c^{\gamma},\left\langle\left\langle s_{\alpha} \cup s_{\alpha}^{\gamma}, F_{\alpha} \cup F_{\alpha}^{\gamma}\right\rangle: \alpha \in A\right\rangle,\left\langle\left\langle t_{\beta} \cup t_{\beta}^{\gamma}, G_{\beta} \cup G_{\beta}^{\gamma}\right\rangle: \beta \in B\right\rangle\right\rangle
$$

and for every $\gamma<\delta<\omega_{1}, \alpha \in A$, and $\beta \in B$, each of $d\left(c^{\gamma}\right) \cap d\left(c^{\delta}\right)$, $d(c) \cap d\left(c^{\gamma}\right), \operatorname{dom}\left(s_{\alpha}^{\gamma}\right) \cap \operatorname{dom}\left(s_{\alpha}^{\delta}\right), F_{\alpha}^{\gamma} \cap F_{\alpha}^{\delta}, \operatorname{dom}\left(t_{\beta}^{\gamma}\right) \cap \operatorname{dom}\left(t_{\beta}^{\delta}\right)$, and $G_{\beta}^{\gamma} \cap G_{\beta}^{\delta}$ is the empty set (this also uses the countability of the sets $H(\xi)$ ).

We now define for all $\gamma<\omega_{1}$,

$$
\begin{aligned}
P(\gamma) & =d\left(c^{\gamma}\right) \cup \bigcup_{\alpha \in A} F_{\alpha}^{\gamma} \cup \bigcup_{\beta \in B} G_{\beta}^{\gamma}, \\
Q(\gamma) & =\bigcup_{\alpha \in A} \operatorname{dom}\left(s_{\alpha}^{\gamma}\right) \cup \bigcup_{\beta \in B} \operatorname{dom}\left(t_{\beta}^{\gamma}\right), \quad \text { and } \\
P & =d(c) \cup \bigcup_{\alpha \in A} F_{\alpha} \cup \bigcup_{\beta \in B} G_{\beta} .
\end{aligned}
$$

It is now easy to find $\gamma<\delta<\omega_{1}$ such that

(i) $\emptyset=P(\gamma) \cap P=P \cap P(\delta)=P(\gamma) \cap P(\delta)$;

(ii) $Q(\gamma) \cap\left\{\zeta<\lambda: \exists \xi \in d\left(c^{\delta}\right) \cup d(c)\left((\xi, \zeta) \in \operatorname{dom}\left(c^{\delta}\right)\right)\right\}=\emptyset$;

(iii) $Q(\delta) \cap\left\{\zeta<\lambda: \exists \xi \in d\left(c^{\gamma}\right) \cup d(c)\left((\xi, \zeta) \in \operatorname{dom}\left(c^{\gamma}\right)\right)\right\}=\emptyset$.

We claim that $p^{\gamma}$ and $p^{\delta}$ are compatible. To see this, note that by (i)-(iii), we can find a $\widehat{c} \supseteq c^{\gamma} \cup c^{\delta}$ such that

$(*)$ if $\xi \in P(\gamma) \cup P$ and $\zeta \in H(\xi) \cap Q(\delta)$, then $\langle\xi, \zeta\rangle \in \operatorname{dom}(\widehat{c})$ and $\widehat{c}(\xi, \zeta)=0$;

$(* *)$ if $\xi \in P(\delta) \cup P$ and $\zeta \in H(\xi) \cap Q(\gamma)$, then $\langle\xi, \zeta\rangle \in \operatorname{dom}(\widehat{c})$ and $\widehat{c}(\xi, \zeta)=0$.

Consider the condition

$$
\begin{aligned}
p=\left\langle\widehat{c},\left\langle\left\langle s_{\alpha} \cup s_{\alpha}^{\gamma} \cup s_{\alpha}^{\delta}, F_{\alpha} \cup F_{\alpha}^{\gamma} \cup F_{\alpha}^{\delta}\right\rangle: \alpha \in A\right\rangle,\right. & \\
& \left.\quad\left\langle\left\langle t_{\beta} \cup t_{\beta}^{\gamma} \cup t_{\beta}^{\delta}, G_{\beta} \cup G_{\beta}^{\gamma} \cup G_{\beta}^{\delta}\right\rangle: \beta \in B\right\rangle\right\rangle ;
\end{aligned}
$$

we show that $p \leq p^{\gamma}\left(p \leq p^{\delta}\right.$ is similar $)$.

Clearly, all inclusion relations are met. Notice that by $(*)$ and $(* *)$ we have for all $\alpha \in A$, all $\zeta \in \operatorname{dom}\left(s_{\alpha}^{\delta}\right)$, and all $\xi \in F_{\alpha} \cup F_{\alpha}^{\gamma}$,

$$
0=\widehat{c}(\xi, \zeta) \leq s_{\alpha}^{\delta}(\zeta), \quad \text { or } \quad \zeta \notin H(\xi),
$$

and for $\beta \in B, \zeta \in \operatorname{dom}\left(t_{\beta}^{\gamma}\right)$, and $\xi \in G_{\beta} \cup G_{\beta}^{\gamma}$,

$$
0=\widehat{c}(\xi, \zeta) \leq t_{\beta}^{\delta}(\zeta), \quad \text { or } \quad \zeta \in H(\xi),
$$

so that $p \leq p^{\gamma}$. This establishes the claim. 
Let $V_{2}$ be the extension of $V_{1}$ by $\mathbb{P}_{1}$; notice that (b) and (c) of the theorem are true by genericity. To complete the proof of the theorem, we need to show:

Claim. $\mathcal{F}$ is unbounded in $V_{2}$.

By way of contradiction, suppose that there is a $\mathbb{P}$-name $\dot{f}$ for an element of $\omega^{\lambda}$ such that $\mathbb{P}_{\mathbb{P}} " \forall \xi<\theta\left(\dot{f}_{\xi} \leq^{+} \dot{f}\right)$ ". (We will see later that $\mathbb{P}$ factors nicely, so if this statement is only forced by some non-trivial condition $p$, we can replace $V_{0}$ with an initial extension obtained from a generic that contains $p$, and then argue as below.)

Assume first $\operatorname{cf}(\lambda) \geq \omega_{1}$ and $\lambda^{\omega} \leq \theta$. Using condition (1) of the function $H$, construct, by recursion on $\gamma<\omega_{1}$, conditions $p^{\gamma}=\left\langle c^{\gamma},\left\langle\left\langle s_{\alpha}^{\gamma}, F_{\alpha}^{\gamma}\right\rangle: \alpha \in\right.\right.$ $\left.\left.A^{\gamma}\right\rangle,\left\langle\left\langle t_{\beta}^{\gamma}, G_{\beta}^{\gamma}\right\rangle: \beta \in B^{\gamma}\right\rangle\right\rangle$, where

$$
c^{\gamma} \Vdash "\left\langle s_{\alpha}^{\gamma}, F_{\alpha}^{\gamma}\right\rangle \in \dot{\mathbb{Q}}_{A_{\alpha}} \text { for } \alpha \in A^{\gamma} \text { and }\left\langle t_{\beta}^{\gamma}, G_{\beta}^{\gamma}\right\rangle \in \dot{\mathbb{R}}_{B_{\beta}} \text { for } \beta \in B^{\gamma "} \text {, }
$$

ordinals $\alpha^{\gamma}<\lambda$ and $\beta^{\gamma}<\theta$, and integers $k^{\gamma}<\omega$ such that if

$$
A(\gamma)=\bigcup_{\alpha \in A^{\gamma}} A_{\alpha} \text { and } B(\gamma)=\bigcup_{\beta \in B^{\gamma}} B_{\beta},
$$

then

(i) if $\operatorname{cf}(\gamma)=\omega$, then $\alpha^{\gamma}=\sup \left\{\alpha^{\delta}: \delta<\gamma\right\}$;

(ii) if $\gamma$ is a successor ordinal, then $\forall \delta<\gamma\left(\alpha^{\gamma}>\alpha^{\delta}\right.$ and $\left.\alpha^{\gamma} \notin A(\delta)\right)$;

(iii) $\forall \delta<\gamma\left(\beta^{\gamma} \notin\left\{\beta^{\delta}\right\} \cup B(\delta) \cup d\left(c^{\delta}\right)\right)$;

(iv) $H\left(\beta^{\gamma}\right) \supseteq\left\{\alpha^{\delta}: \delta<\gamma\right\}$; and

(v) $p^{\gamma} \Vdash_{\mathbb{P}} " \forall \zeta<\lambda\left(\dot{f}_{\beta^{\gamma}}(\zeta) \leq k^{\gamma}\right.$ or $\left.\dot{f}_{\beta \gamma}(\zeta) \leq \dot{f}(\zeta)\right)$ ".

To avoid having to work with names, we are again assuming that the $\mathbb{P}_{0}$ part of a condition is strong enough to decide the $\mathbb{P}_{1}$ part. Also notice that by conditions (i) and (ii) of the recursion, $S_{0}=\left\{\alpha^{\gamma}: \gamma<\omega_{1}\right\}$ is club in $\alpha^{\prime}=\sup \left\{\alpha^{\gamma}: \gamma<\omega_{1}\right\}$. Thus $S \subseteq S_{0}$ is stationary in $\alpha^{\prime}$ if and only if $\widetilde{S}=\left\{\gamma<\omega_{1}: \alpha^{\gamma} \in S\right\}$ is stationary in $\omega_{1}$.

We now define regressive functions $a, b, c: \omega_{1} \rightarrow\left[\omega_{1}\right]^{<\omega}$ and $k: \omega_{1} \rightarrow \omega$ by:

$$
\begin{aligned}
& a(\gamma)=\left\{\delta<\gamma:\left(A^{\gamma} \cap A^{\delta}\right) \backslash \bigcup_{\varepsilon<\delta} A^{\varepsilon} \neq \emptyset\right\} ; \\
& b(\gamma)=\left\{\delta<\gamma:\left(B^{\gamma} \cap B^{\delta}\right) \backslash \bigcup_{\varepsilon<\delta} B^{\varepsilon} \neq \emptyset\right\} ; \\
& c(\gamma)=\left\{\delta<\gamma:\left(d\left(c^{\gamma}\right) \cap d\left(c^{\delta}\right)\right) \backslash \bigcup_{\varepsilon<\delta} d\left(c^{\varepsilon}\right) \neq \emptyset\right\} ; \text { and } \\
& k(\gamma)=k^{\gamma} .
\end{aligned}
$$


By Fodor's lemma, we can find $\Delta_{a}, \Delta_{b}, \Delta_{c} \in\left[\omega_{1}\right]^{<\omega}$, a $k_{0} \in \omega$, and a stationary $\widetilde{S}_{1} \subseteq \omega_{1}$ such that for $\gamma \in \widetilde{S}_{1}, a(\gamma)=\Delta_{a}, b(\gamma)=\Delta_{b}, c(\gamma)=\Delta_{c}$ and $k(\gamma)=k_{0}$.

Set $\bar{A}=\bigcup_{\gamma \in \Delta_{a}} A(\gamma)$ and $\bar{B}=\bigcup_{\gamma \in \Delta_{b}} B(\gamma) \cup \bigcup_{\gamma \in \Delta_{c}} d\left(c^{\gamma}\right)$. Then for $\gamma \neq \gamma^{\prime} \in \widetilde{S}_{1}$, we have

(a) $\left(A^{\gamma} \cap A^{\gamma^{\prime}}\right) \backslash \bigcup_{\delta \in \Delta_{a}} A^{\delta}=\emptyset$;

(b) $\left(B^{\gamma} \cap B^{\gamma^{\prime}}\right) \backslash \bigcup_{\delta \in \Delta_{b}} B^{\delta}=\emptyset$; and

(c) $\left(d\left(c^{\gamma}\right) \cap d\left(c^{\gamma^{\prime}}\right)\right) \backslash \bar{B}=\emptyset$.

We now factor $\mathbb{P}_{0}$ as $\mathbb{P}_{0}^{0} \times \mathbb{P}_{0}^{1}$, where

$$
\mathbb{P}_{0}^{0}=\left\{c \in \mathbb{P}_{0}: \forall \zeta<\lambda \forall \xi<\theta((\xi, \zeta) \in \operatorname{dom}(c) \Rightarrow \xi \in \bar{B} \vee \zeta \in \bar{A})\right\}
$$

and

$$
\mathbb{P}_{0}^{1}=\left\{c \in \mathbb{P}_{0}: \forall \zeta<\lambda \forall \xi<\theta((\xi, \zeta) \in \operatorname{dom}(c) \Rightarrow \xi \notin \bar{B} \wedge \zeta \notin \bar{A})\right\} .
$$

In turn, if we set $A=\bigcup_{\delta \in \Delta_{a}} A^{\delta}, B=\bigcup_{\delta \in \Delta_{b}} B^{\delta}$,

$$
\mathbb{P}_{1}^{0}=\prod_{\alpha \in A} \mathbb{Q}_{A_{\alpha}} \times \prod_{\beta \in B} \mathbb{R}_{B_{\beta}}, \quad \text { and } \quad \mathbb{P}_{1}^{1}=\prod_{\alpha \notin A} \mathbb{Q}_{A_{\alpha}} \times \prod_{\beta \notin B} \mathbb{R}_{B_{\beta}},
$$

then $\mathbb{P}$ can be factored as

$$
\mathbb{P}=\left(\mathbb{P}_{0}^{0} \star \dot{\mathbb{P}}_{1}^{0}\right) \star\left(\mathbb{P}_{0}^{1} \star \dot{\mathbb{P}}_{1}^{1}\right) .
$$

Let $G^{0}$ be $\mathbb{P}^{0}=\mathbb{P}_{0}^{0} \star \dot{\mathbb{P}}_{1}^{0}$-generic over $V_{0}$, and let $V^{0}=V_{0}\left[G^{0}\right]$. In $V^{0}$, set $\mathbb{P}^{1}=\mathbb{P}_{0}^{1} \star \dot{\mathbb{P}}_{1}^{1}$.

We claim that $G^{0}$ can be chosen so that $\widetilde{S}^{0}=\left\{\gamma \in \widetilde{S}_{1}: p^{\gamma} \uparrow \mathbb{P}^{0} \in G^{0}\right\}$ is a stationary subset of $\omega_{1}$ in $V^{0}$. Otherwise, we can find a $\mathbb{P}^{0}$-name $\dot{C}$ for a club subset of $\omega_{1}$ such that $\Vdash_{\mathbb{P}^{0}} " \forall \gamma<\omega_{1}\left(\gamma \in \dot{C} \Rightarrow p^{\gamma} \uparrow \mathbb{P}^{0} \notin \dot{G}^{0}\right)$ ". Because $\mathbb{P}^{0}$ is ccc, we can find a club $C_{0} \in V_{0}$ such that $\Vdash_{\mathbb{P}^{0}}$ " $C_{0} \subseteq \dot{C}$ ". Take a $\gamma \in C_{0} \cap \widetilde{S}_{1} ;$ then

$$
p^{\gamma} \uparrow \mathbb{P}^{0} \Vdash_{\mathbb{P}^{0}} " p^{\gamma} \uparrow \mathbb{P}^{0} \in \dot{G}^{0} \text { and } \gamma \in \dot{C} ",
$$

a contradiction.

Let $S^{0}=\left\{\alpha^{\gamma}: \gamma \in \widetilde{S}^{0}\right\}$. For $\gamma \in \widetilde{S}^{0}$, define

$$
e\left(\alpha^{\gamma}\right)=\max \left\{\zeta<\alpha^{\gamma}:\left(\beta^{\gamma}, \zeta\right) \in \operatorname{dom}\left(c^{\gamma}\right)\right\} .
$$

(When we are talking about $c^{\gamma}$ (or other parts of conditions), we really mean $c^{\gamma} \uparrow \mathbb{P}_{0}^{1}$ - this is ok because we have chosen $c^{\gamma}$ so that $c^{\gamma} \uparrow \mathbb{P}_{0}^{0} \in G^{0}$.) Notice that $e$ is regressive on $S^{0}$, so there is a stationary $T^{0} \subseteq S^{0}$ and a $\zeta_{0}<\alpha^{\prime}$ so that $\forall \gamma \in T^{0}\left(e(\gamma)=\zeta_{0}\right)$. Set $\widetilde{T}^{0}=\left\{\gamma<\omega_{1}: \alpha^{\gamma} \in T^{0}\right\}$.

Now choose $\delta_{0}<\omega_{1}$ so that $\alpha^{\delta_{0}} \notin \bar{A}$ and $\alpha^{\delta_{0}}>\zeta_{0}$. Recall that $\alpha^{\delta_{0}} \in$ $H\left(\beta^{\gamma}\right)$ whenever $\gamma>\delta_{0}$ and $\gamma \in \widetilde{T}^{0}$. Also notice that without loss of generality $\beta^{\gamma} \notin \bar{B}$ for $\gamma \in \widetilde{T}^{0}$. Let $\dot{G}^{1}$ be the canonical name for a $\mathbb{P}^{1}$-generic 
filter over $V^{0}$. We claim that

$$
\Vdash_{\mathbb{P}^{1}} \cdot \forall k \in \omega \exists \gamma \in \widetilde{T}^{0}\left(p^{\gamma} \in \dot{G}^{1} \wedge \dot{f}_{\beta^{\gamma}}\left(\alpha^{\delta_{0}}\right)=k\right) .
$$

To see this, suppose that $p^{1} \in \mathbb{P}^{1}$ and $k \in \omega$. Say

$$
p^{1}=\left\langle c^{1},\left\langle\left\langle s_{\alpha}^{1}, F_{\alpha}^{1}\right\rangle: \alpha \in A^{1}\right\rangle,\left\langle\left\langle t_{\beta}^{1}, G_{\beta}^{1}\right\rangle: \beta \in B^{1}\right\rangle\right\rangle .
$$

By conditions (a)-(c), we can find a $\gamma \in \widetilde{T}^{0}$ such that the following intersections are all empty: $d\left(c^{1}\right) \cap\left(d\left(c^{\gamma}\right) \cup\left\{\beta^{\gamma}\right\}\right), A^{1} \cap A^{\gamma}$, and $B^{1} \cap B^{\gamma}$.

Set $p_{0}^{\gamma}=\left\langle c^{\gamma} \cup\left\{\left\langle\left(\beta^{\gamma}, \alpha^{\delta_{0}}\right), k\right\rangle\right\},\left\langle\left\langle s_{\alpha}^{\gamma}, F_{\alpha}^{\gamma}\right\rangle: \alpha \in A^{\gamma}\right\rangle,\left\langle\left\langle t_{\beta}^{\gamma}, G_{\beta}^{\gamma}\right\rangle: \beta \in B^{\gamma}\right\rangle\right\rangle$; then $p^{1} \cup p_{0}^{\gamma}$ is a condition extending both $p^{1}$ and $p_{0}^{\gamma}$ that forces $\dot{f}_{\beta^{\gamma}}\left(\alpha^{\delta_{0}}\right)=k$. This establishes $(*)$.

Let $G^{1}$ be $\mathbb{P}^{1}$-generic over $V^{0}$, and set $V^{1}=V^{0}\left[G^{1}\right]=V_{2}$. Let $k_{1}=$ $\dot{f}\left[G^{1}\right]\left(\alpha^{\delta_{0}}\right)$. By $(*)$, there is a $k \in \omega$ and a $\gamma \in \widetilde{T}^{0}$ so that $k>k_{0}, k_{1}$ and $\dot{f}_{\beta^{\gamma}}\left[G^{1}\right]\left(\alpha^{\delta_{0}}\right)=k$. This contradicts the fact that each $\dot{f}_{\beta^{\gamma}}\left[G^{1}\right](\zeta)$ is forced to be less than either $k_{0}$ or $\dot{f}\left[G^{1}\right](\zeta)$. This establishes the claim and the theorem in most cases.

In case $\lambda$ is regular and $\lambda^{\omega} \leq \theta$ fails, we use conditions (2) and (3) of the function $H$ to carry out a similar construction, replacing (ii) and (iv) by

(ii) $)^{\prime} \forall \delta<\gamma\left(\alpha^{\gamma}>\max \left\{\alpha^{\delta}, \sup (A(\delta))\right\}\right)$; and

(iv) ${ }^{\prime} \sup \left(H\left(\beta^{\gamma}\right)\right)=\alpha^{\gamma}$ (and thus $\operatorname{cf}\left(\alpha^{\gamma}\right)=\omega$ ).

The rest of the argument is very similar to the first case, and we leave it to the reader to figure out the details.

In case $\operatorname{cf}(\lambda)=\omega$, we write $\lambda=\bigcup_{n} \lambda_{n}$ where $\lambda_{n}<\lambda_{n+1}<\lambda$ and the $\lambda_{n}$ are regular. We again do a similar construction, this time producing ordinals $\alpha_{n}^{\gamma}<\lambda_{n}$; (i), (ii) and (iv) are generalized to

(i)" if $\operatorname{cf}(\gamma)=\omega$, then $\alpha_{n}^{\gamma}=\sup \left\{\alpha_{n}^{\delta}: \delta<\gamma\right\}$ for all $n$;

(ii) " if $\gamma$ is a successor ordinal, then $\forall n \forall \delta<\gamma\left(\alpha_{n}^{\gamma}>\alpha_{n}^{\delta}\right.$ and, if $A(\delta) \cap \lambda_{n}$ is bounded in $\lambda_{n}$, then $\left.\alpha_{n}^{\gamma}>\sup (A(\delta))\right)$; and

(iv) $)^{\prime \prime} H\left(\beta^{\gamma}\right) \supseteq\left\{\alpha_{n}^{\delta}: n \in \omega \wedge \delta<\gamma\right\}$.

The proof continues as before. Notice that there must be $n \in \omega$ so that $\bar{A} \cap\left\{\alpha_{n}^{\gamma}: \gamma<\omega_{1}\right\}$ is bounded in $\left\{\alpha_{n}^{\gamma}: \gamma<\omega_{1}\right\}$. We complete the argument with all $\alpha^{\gamma}$ replaced by $\alpha_{n}^{\gamma}$.

4. Topological consequences. As shown in [LL], the existence of a $(\theta, \lambda)$-good set is equivalent to the existence of a first countable $<\theta$-cwH space $X$ with a closed discrete set $D$ of cardinality $\theta$ such that $D$ is not separated and

$$
\begin{aligned}
\lambda=\min \{|E|: E \subseteq D, D \backslash E \text { is separated, and } \\
\left.\qquad F \in[E]^{<|E|}((D \backslash E) \cup F \text { is separated })\right\} .
\end{aligned}
$$


By these results, Theorem 3.5 gives a new example of a first countable space in which cwH fails for the first time at a singular cardinal. Notice that this space is easier to construct than the examples in [FS] and [K], and can be made $\mathrm{cwH}$ by removing a countable closed discrete set.

Suppose that $\lambda<\theta$ are uncountable cardinals that satisfy the hypotheses of Theorem 3.6. The $(\theta, \lambda)$-good set constructed by Theorem 3.6 gives a new consistent example of a first countable, $<\theta-\mathrm{cwH}$ space $X$ that is not $\leq \theta-\mathrm{cwH}$. The set of non-isolated points of $X$ is the union of two disjoint closed discrete sets $D$ and $E$, where $|D|=\theta,|E|=\lambda$, both $D$ and $E$ are separated, but $D$ and $E$ are not contained in disjoint open sets. Thus, $X$ can be made cwH by removing the small closed discrete set $E$.

When $\lambda=\theta$ satisfy the hypotheses of Theorem 3.6 and are singular, the space $X$ obtained resembles the first countable $<\theta$-cwH not $\leq \theta$-cwH space constructed in [FS], though the models in which the constructions take place may be quite different. In both spaces, the set of non-isolated points is the union of two disjoint closed discrete sets of cardinality $\theta$, each of which is separated, but that are not contained in a pair of disjoint open sets.

When $\theta$ is singular and greater than $\lambda^{\omega}$, we obtain a first countable space in which $\mathrm{cwH}$ fails for the first time at $\theta$, yet the space can be made $\mathrm{cwH}$ by removing a closed discrete set of cardinality $\lambda$.

5. Questions. We have shown for many cardinals $\lambda \leq \theta$ that "there is a $(\theta, \lambda)$-good set" is consistent. On the other hand, under $\mathrm{GCH}$, there are no $(\theta, \lambda)$-good sets when $\omega_{1} \leq \operatorname{cf}(\lambda) \leq \lambda<\theta$, so for these cardinals, the existence of a $(\theta, \lambda)$-good set is independent of ZFC. There are no $(\theta, \theta)$-good sets when $\theta$ is singular of countable cofinality (see [LL]), so we ask:

(1) Is it consistent to have a $(\theta, \lambda)$-good set when $\operatorname{cf}(\theta)=\omega$ and $\omega_{1} \leq$ $\operatorname{cf}(\lambda) \leq \lambda<\theta$ ?

(2) Suppose that $\omega=\operatorname{cf}(\lambda)<\lambda$. Is there, in ZFC, a cardinal $\theta$ such that $\lambda<\theta \leq \lambda^{\omega}$ and there is a $(\theta, \lambda)$-good set?

Of course, the most important question, originally asked by Dow and Todorčević, is:

(3) Does ZFC imply the existence of an $\left(\omega_{2}, \omega_{2}\right)$-good set?

Todorčević [T] showed that $\square\left(\omega_{2}\right)$ implies that there is a $\left(\omega_{2}, \omega_{2}\right)$-good set. So at least a weakly compact cardinal is required to produce a model with no $\left(\omega_{2}, \omega_{2}\right)$-good sets. Fleissner used $E_{\omega_{2}}^{\omega}$ (i.e., "there is a non-reflecting stationary subset of $\omega_{2}$ consisting of ordinals of countable cofinality") to construct a first countable, $<\omega_{2}-\mathrm{cwH}$ space that is not $\leq \omega_{2}-\mathrm{cwH}$, so $E_{\omega_{2}}^{\omega}$ can also be used to produce an $\left(\omega_{2}, \omega_{2}\right)$-good set.

Recall that Beaudoin (and independently, Magidor) showed that PFA 
is consistent with $E_{\omega_{2}}^{\omega}$, while $\mathrm{PFA}^{+}$implies that stationary sets reflect. We conclude with:

(4) Does $\mathrm{PFA}^{+}$imply that there are no $\left(\omega_{2}, \omega_{2}\right)$-good sets?

\section{References}

[Br] J. Brendle, notes.

[FS] W. G. Fleissner and S. Shelah, Incompactness at singulars, Topology Appl. 31 (1989), 101-107.

[G] G. Gruenhage, $k$-spaces and products of closed images of metric spaces, Proc. Amer. Math. Soc. 80 (1980), 477-482.

[H] F. Hausdorff, Die Graduierung nach dem Endverlauf, Abh. Königl. Sächs. Gesell. Wiss. Math.-Phys. Kl. 31 (1909), 296-334.

[J] H. Judah, private communication.

[K] P. Koszmider, Kurepa trees and topological non-reflection, preprint.

$[\mathrm{Ku}] \mathrm{K}$. Kunen, Inaccessibility properties of cardinals, Ph.D. dissertation, Stanford, 1968.

[LL] T. LaBerge and A. Landver, Tightness in products of fans and psuedo-fans, Topology Appl. 65 (1995), 237-255.

[R] F. Rothberger, Sur les familles indénombrables de suites de nombres naturels et les problèmes concernant la propriété C, Proc. Cambridge Philos. Soc. 37 (1941), $109-126$.

[T] S. Todorčević, My new fan, preprint.

Department of Mathematics

University of Tübingen

Tübingen, Germany

E-mail:

jobr@michelangelo.mathematik.uni-tuebingen.de

Department of Mathematics

Dartmouth College

Hanover, New Hampshire 03755

U.S.A.

E-mail: Jorg.Brendle@Dartmouth.edu
Department of Mathematics Union College Schenectady, New York 12308 U.S.A.

E-mail: laberget@unvax.union.edu Department of Mathematical Sciences Northern Illinois University DeKalb, Illinois 60115 U.S.A.

E-mail: laberget@math.niu.edu

Received 14 June 1994;

in revised form 29 February 1996 